\title{
B cells and complement at the forefront of chemotherapy
}

Catherine Sautès-Fridman*, Lubka Roumenina

Centre de Recherche des Cordeliers, INSERM, Sorbonne Université, Université de Paris, Team inflammation, complement and cancer, F-75006, Paris, France

*corresponding author: Catherine Sautès-Fridman, Catherine.fridman@crc.jussieu.fr

Efficient anti-cancer therapies activate anti-tumor immunity, particularly borne by $T$ cells in the tumor microenvironment. We discuss here the implication of $B$ cells and Tertiary Lymphoid Structures in the context of chemotherapy-provoked complement activation resulting in the induction of a $B$ cell subset that modulates $T$ cell activities.

Chemotherapeutic agents such as anthracyclines, cyclophosphamide, oxaliplatin and taxanes commonly used to treat breast cancer $(\mathrm{BC})$ not only increase the immunogenicity of tumor cells by inducing Immunogenic Cell Death (ICD) but also modify the tumor microenvironment (TME). Chemotherapies may favor the anti-cancer immune responses by inducing the production of $\mathrm{T}$ cell attracting chemokines and by increasing the $\mathrm{CD} 8 / \mathrm{Treg}^{\mathrm{ratio}}{ }^{1}$. The mechanistic concept behind the therapeutic responses to immune checkpoint blockade (ICB) therapies with antibodies against PD-1/PD-L1 or CTLA-4 in patients with cancer relies also on modification of T cells, to alleviate their inhibition or trigger their expansion.

In a recent paper, Lu et al. ${ }^{2}$ surprisingly bring B cells and complement at the forefront of the mechanisms sustaining the immunomodulatory effect of chemotherapy on the TME. These authors show that, to be efficient, chemotherapy relies on the induction of an ICOS-L+ B cells subset expressing the complement receptor CR2 and subsequently leading to an increase of the $C D 8 /$ Treg ratio. Emergence of this $B$ cell subset is associated with improved therapeutic 
efficacy of neo-adjuvant chemotherapy in BC, particularly in triple negative tumors, and with prolonged survival of the patients. This work also highlights how complement components orchestrate modulation of B cells in TME. Complement component C3 activation on dying cells and interaction with CR2 induces the generation of ICOS-L+B cells (Figure). On the contrary, CD55, a cell surface complement regulatory protein, allows tumors cells to escape by inhibiting complement activation. Using mice genetically deficient in ICOS-L, C3 and CR2, Lu et al. found that these events are necessary for the generation of anti-tumor immunity induced by doxorubicin. They did not investigate the role of antibodies produced by intratumoral B cells. However, intratumoral complement activation on IgG deposits may occur as reported in patients with clear cell renal cell cancer, where it was shown to have a deleterious impact on survival ${ }^{3,4}$. In most mouse models of cancer as well (except BC) complement is deleterious in absence of treatment, but becomes necessary for the antitumoral effect in case of adoptive T cell therapy with tumor-specific T cells (reviewed in ${ }^{4}$ ). Further studies are needed to find out to what extend the role of CD55 on tumor cells and of ICOS-L+B cells is applicable to other cancers and other contexts.

In human cancers, B cells have been identified in many tumor types and were found to be associated with good or neutral prognosis in most of them ${ }^{5}$. The $B$ cells are principally enriched in the invasive margin, most of them being present in Tertiary Lymphoid Structures (TLS). TLS are sites of generation of both T and B cells immunity toward antigens present in the tissue where they develop. Thus, TLS may allow presentation of tumor antigens to T and B cells, triggering $T$ and $B$ cell activation, proliferation and differentiation into effector cells (cytotoxic and helper T cells, and antibody secreting cells) as well as activation of memory T 
and $B$ cells activation in a similar way to secondary lymphoid organs ${ }^{6}$. Antigen presentation by $B$ cells to $T$ follicular helper cells (Tfh) occurs in B cell follicles, leading to B cell activation and maturation in short lived plasma cells. Follicular B cells (FO B cells) can also undergo further affinity maturation and isotype switch into germinal centers (GCs) to become long lived plasma cells or memory B cells. Complement and CR2 play a major role in these processes, allowing the CR2 dependent transfer of immune complexes consisting of IgM or IgG, and C3 degradation products from macrophages to CR2+ FO B cells first and then onto Fc $\gamma \mathrm{R}+; \mathrm{CR} 1+, \mathrm{CR} 2+$ follicular dendritic cells (FDC) in the GC, favoring presentation of antigens to $B$ cells at every step ${ }^{7}$. ICOS-ICOS-L interactions occurring between $T$ and $B$ cells are decisive as well, patients with ICOS deficiency having severely reduced circulating Tfh and major defects in antibody generation.

Strikingly, Lu et $\mathrm{al}^{2}$ observe an induction of tumor-associated TLS after neo-adjuvant chemotherapy, particularly in responding patients. These data are reminiscent of the three very recent reports showing that in patients treated with ICB, intratumoral B cells and TLS strongly correlate with response ${ }^{8,9}$. In sarcoma patients, B cells and TLS are predictive of ICB response regardless of the intratumoral $\mathrm{T}$ cell content ${ }^{8}$. In melanoma Helmink et al. also showed that the predictive value of B cell was independent of T cell density ${ }^{9}$ while Cabrita et al found that the high predictive value of B cells requires high intratumoral T cell contents ${ }^{9}$. In addition, B cells and TLS have been associated with longer survival in TNBC ${ }^{10}$. Altogether these studies and the paper by Lu et al. point to the fact that B lymphocytes, probably when activated in TLS, are a focus for impact for not only ICB but also chemotherapy (Figure).

One key question is how tumor infiltrating B cells exert their prognostic and predictive 
impact. Tumors contain a majority of memory B cells which may cooperate with CD4+Th cells to facilitate the activation of CD8+ cytotoxic T cells. B cells or plasma cells could also cross present tumor antigens to CD8+T cells, as suggested by the higher prognostic effect of CD8+T cells when B cells or plasma cells are present ${ }^{5}$. The work of Lu et al. suggest that the ICOS-L+ B cells subset emerging after chemotherapy elicit anti-tumor immunity by increasing the ratio of $\mathrm{T}$ effector cells (CD8+ cytotoxic $\mathrm{T}$ cells and CD4+Th1 cells) to $\mathrm{CD} 4+\mathrm{T}$ regulatory cells. However, B cells functions extend much beyond, notably via the production of antibodies against tumor associated antigens that may sensitize T cells through IC uptake by dendritic cells, a process likely to occur within TLS or destroy tumor cells via antibodydependent cytotoxicity (ADCC) or complement-dependent cytotoxicity $(C D C)^{6}$.

References

1. Fridman, W. H., Zitvogel, L., Sautès-Fridman, C. \& Kroemer, G. The immune contexture in cancer prognosis and treatment. Nature Reviews Clinical Oncology 14, 717734 (2017).

2. Lu, Y. et al. Complement Signals Determine Opposite Effects of B Cells in Chemotherapy-Induced Immunity. Cell (2020) doi:10.1016/j.cell.2020.02.015.

3. Roumenina, L. T. et al. Tumor Cells Hijack Macrophage-Produced Complement C1q to Promote Tumor Growth. Cancer Immunol Res 7, 1091-1105 (2019).

4. Roumenina, L. T., Daugan, M. V., Petitprez, F., Sautès-Fridman, C. \& Fridman, W. H. Context-dependent roles of complement in cancer. Nat. Rev. Cancer 19, 698-715 (2019).

5. Wouters, M. C. A. \& Nelson, B. H. Prognostic Significance of Tumor-Infiltrating B Cells and Plasma Cells in Human Cancer. Clin Cancer Res 24, 6125-6135 (2018).

6. Sautès-Fridman, C., Petitprez, F., Calderaro, J. \& Fridman, W. H. Tertiary lymphoid structures in the era of cancer immunotherapy. Nat. Rev. Cancer 19, 307-325 (2019).

7. Kranich, J. \& Krautler, N. J. How Follicular Dendritic Cells Shape the B-Cell Antigenome. Front Immunol 7, 225 (2016).

8. Petitprez, F. et al. B cells are associated with survival and immunotherapy response in sarcoma. Nature (2020) doi:10.1038/s41586-019-1906-8.

9. Bruno, T. C. New predictors for immunotherapy responses sharpen our view of the tumour microenvironment. Nature 577, 474-476 (2020).

10. Garaud, S. et al. Tumor infiltrating B-cells signal functional humoral immune responses in breast cancer. JCI Insight 5, (2019). 
Acknowledgements : We wish to thank W.H. Fridman for discussions about this topic and review of this manuscript.

Figure : How tertiary lymphoid structures may orchestrate chemotherapy-induced immunity Chemotherapy induces tumor cell death and complement component C3 cleavage, local inflammation, triggering formation of TLS where immune response is activated locally though antigen presentation by antigen presenting cells, including B cells. Activation of follicular B cells by C3 complement cleavage products via CR2 induces the emergence of an ICOS+ B cell subset which activates ICOS-L+ T cells and finally leads to increased CD8+ cytotoxic and CD4+Th1 effector cells. Complement cleavage products may also facilitate affinity maturation of B cells through binding to IC on follicular dendritic cells in GCs.
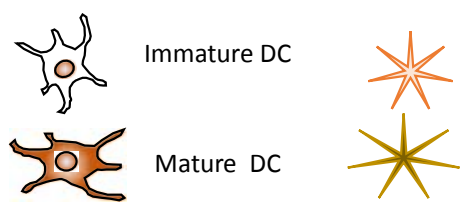

Fibroblastic reticular cell
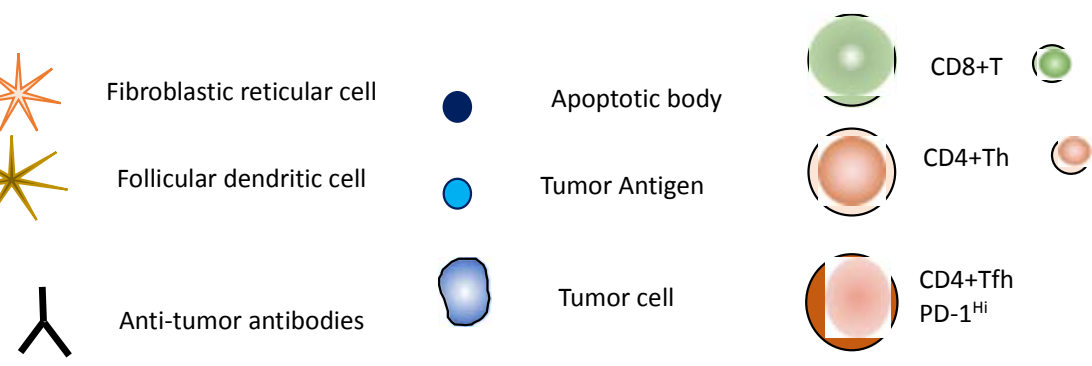

Anti-tumor antibodies

Tumor cell

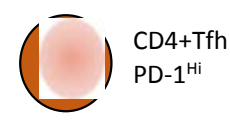

$$
\begin{aligned}
& \text { C3 cleavage } \\
& \text { products }
\end{aligned}
$$

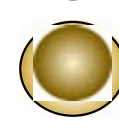

$\mathrm{CD} 20+\mathrm{B}$

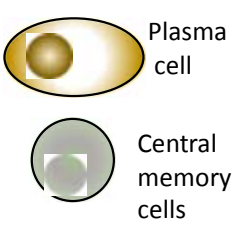

